\title{
Coupling of high-quality-factor optical resonators
}

\author{
Patrice Salzenstein ${ }^{1}$, Michel Mortier ${ }^{2}$, Hélène Sérier-Brault ${ }^{2}$, \\ Rémi Henriet $^{1}$, Aurélien Coillet ${ }^{1}$, Yanne K Chembo ${ }^{1}$, \\ Alphonse Rasoloniaina ${ }^{3}$, Yannick Dumeige ${ }^{3}$ and Patrice Féron ${ }^{3}$
}

\author{
${ }^{1}$ Centre National de la Recherche Scientifique (CNRS), Franche Comté Electronique Thermique Optique \\ Sciences et Technologies (FEMTO-ST), 32 avenue de l'Observatoire, F-25044 Besançon Cedex, France \\ ${ }^{2}$ Centre National de la Recherche Scientifique (CNRS), LCMCP, Chimie ParisTech, 11, rue Pierre et \\ Marie Curie, F-75231 Paris Cedex 05, France \\ ${ }^{3}$ Enssat-Foton, 6, rue de Kerampont, BP 80518, F-22305 Lannion Cedex, France \\ E-mail: patrice.salzenstein@femto-st.fr
}

Received 6 September 2012

Accepted for publication 7 February 2013

Published 15 November 2013

Online at stacks.iop.org/PhysScr/T157/014024

\begin{abstract}
We improve theoretically and experimentally the problem of the coupling between a high $Q$-factor resonator and its external coupler. We have observed oscillations of ringing induced by the sweeping of the excitation frequency of an active microsphere. Thanks to this approach, the quality factor of an optical resonator was measured and we obtained $Q=5.8 \times 10^{8}$.
\end{abstract}

PACS numbers: 42.60.Da, 85.60.-q, 06.20.fb, 89.20.Kk, 42.82.Bq, 42.82.Gw

(Some figures may appear in colour only in the online journal)

\section{Introduction}

Optical resonators with high quality factor ( $Q$-factor) present great interest for several applications in optoelectronic [1], metrology or fundamental physics [2, 3]. For instance, they can be used in optical filtering, all optical switching, low-threshold nonlinear optics, etc. Main results have been obtained since 1996 [4]. Recently, results have been obtained with mono-crystalline resonators such as $\mathrm{MgF}_{2}$, quartz or $\mathrm{CaF}_{2}$ whispering gallery mode (WGM) resonators [5-9]. Rare-earth element-doped microspheres are tiny lasers that intrinsically offer narrow linewidth emission [10]. A key feature of the characterization of the resonators is to perform good coupling. Performing this characterization is quite challenging because it is a necessary but difficult technique. That is why we improve theoretically and experimentally the problem of the coupling between a high- $Q$-factor resonator and its external coupler. They are characterized by different methods such as slow and fast frequency sweeping [11]. Resonators can be coupled to tapered fibers or prisms. We focus here on characterization techniques of passive optical resonators or active (erbium-doped ZBLALiP [12, 13]) WGM resonators in terms of $Q$-factor determination.

\section{Properties of a doped microresonator}

Figure 1 shows schematically the WGM microresonator and its access line. It is possible to isolate each resonance, although the resonator is inherently multimode. We can work with each resonance separately. The studied resonator is then considered to be single mode.

A mode with an amplitude $a(t)$ is characterized by the lifetime of the field $\tau_{0}$ limited by losses, by the coupling rate of the access line that is proportional to $1 / \tau_{\mathrm{e}}$ and by the angular frequency of the resonance $\omega_{0}$. The amplitude of the input signal is denoted as $s_{\text {in }}(t)$; we can then calculate the amplitude of the output signal $s_{\text {out }}(t)=-s_{\text {in }}(t)+\sqrt{2 / \tau_{\mathrm{e}}} a(t)$ by integration:

$$
\frac{\mathrm{d} a}{\mathrm{~d} t}=\left(\mathrm{j} \omega_{0}-\frac{1}{\tau}\right) a(t)+\sqrt{\frac{2}{\tau_{\mathrm{e}}}} s_{\mathrm{in}}(t) .
$$

In equation (1), $\tau=\left(1 / \tau_{0}+1 / \tau_{\mathrm{e}}\right)^{-1}$ is the lifetime of the field in the cavity related to factor $Q=\omega_{0} \tau / 2$. We assume that the input signal is harmonic $s_{\text {in }}(t)=S_{\text {in }} \mathrm{e}^{\mathrm{j} \omega t}$ and the output signal is $s_{\text {out }}(t)=S_{\text {out }} \mathrm{j}^{\mathrm{j} \omega t}$. As a consequence, the transfer 


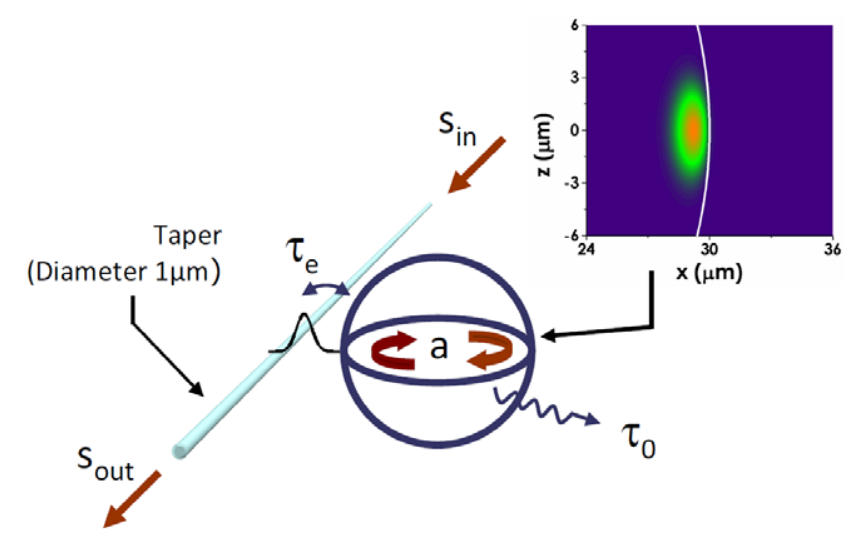

Figure 1. Schematic representation of the microresonator and its access line.

function is given by

$$
T(\delta)=\left|\frac{s_{\text {out }}}{s_{\text {in }}}\right|^{2}=\frac{\left(1 / \tau_{\mathrm{e}}-1 / \tau_{0}\right)^{2}+4 \pi^{2} \delta^{2}}{\left(1 / \tau_{\mathrm{e}}+1 / \tau_{0}\right)^{2}+4 \pi^{2} \delta^{2}} .
$$

In this expression $\delta$ is the frequency mismatch given by $\omega=\omega_{0}+2 \pi \delta$. Different configurations are possible according to the relative value of $\tau_{\mathrm{e}}$ and $\tau_{0}$. If $\tau_{0} \rightarrow \infty$ then the resonator has no losses, so $T(\delta)=1$ as illustrated in figure 1(b). But if we suppose that $\tau_{\mathrm{e}}=\tau_{0}$, then the resonator is in critical coupling and transmission at the resonance $T(0)$ becomes zero. If $\tau_{0}<-\tau_{\mathrm{e}}$, the resonator presents some gain (even if it stays less than the laser threshold) and it behaves like an amplifier; then $T(0)>1$ [14].

\section{Measurements}

Amplification measurements were performed in erbium-doped ZBLALiP microspheres. Diameters of the microspheres are between 50 and $150 \mu \mathrm{m}$. These spheres are obtained by fusion in a microwave inductively coupled plasma [10]. The usual measurement method consists in sweeping the frequency of a laser emitting around $1550 \mathrm{~nm}$, in order to obtain a description of one resonance for the microsphere. Simultaneously, this microsphere is pumped by a laser at $1480 \mathrm{~nm}$ propagating in the opposite direction, as described in figure 2 .

Thanks to the pumping, the sphere delivers a laser signal for one or several resonances far from the studied resonance: it helps to stabilize the gain. An optical spectrum analyzer helps in controlling the microsphere laser emission. To illustrate how this technology can be applied, the laser frequency is swept slowly enough to stay in stationary approximation, to describe a narrow resonance as illustrated in figure 3.

Our approach is different. The analysis begins by rapidly sweeping the frequency of the laser. This process is analogous to what occurs when the speed is slow, but provides definitely an easier and unquestionably precise determination of the $Q$-factor. This approach is implemented by utilizing the following process. This allows us to work in the ringing regime of the microsphere. It has already been demonstrated that it is possible to determine the characteristic parameters of the microsphere [11, 15] if we compare the profile of the ringing oscillations to an analytical model obtained thanks to equation (1) and to an incident field like $s_{\text {in }}(t)=S_{\text {in }} \mathrm{e}^{\mathrm{j}\left(\omega_{i}+\pi V_{s^{t}}\right) t}$. The sweeping speed of the laser, $V_{\mathrm{S}}$, is often difficult to obtain precisely. But it is directly obtained by the method described above. The results are illustrated in figure 4 , where two time profiles are obtained on the same resonance, with two different speeds (the second one is two times faster) for sweeping the laser. While the deduced speeds are in a good ratio, adjustment provides almost the same results for $\tau_{0}$ and $\tau_{\mathrm{e}}$. Observed differences for $\tau_{0}$ and $\tau_{\mathrm{e}}$ are mainly due to the fluctuations of the taper position. It has an influence on the signal coupling, and also on the pump. When performing the adjustment from the obtained ringing oscillation profiles, it has to be underlined that we deduce a quality factor as high as $Q=5.8 \times 10^{8}$. The analysis proceeds by matching the theoretical and experimental curves. $Q$-factor estimation may be quickly obtained, and the efficiency of such a fast sweeping is really good. This is the desired result, which gives great insight into the resonator coupling and $Q$-factor estimation problem.

\section{Coupling with a passive resonator and integration into an oscillator}

An optoelectronic oscillator (OEO) with an optical resonator coupled to an optical fiber delivers a free spectral range microwave signal [1]. To implement this OEO in terms of miniaturization, it is possible to use a high WGM resonator with magnesium fluoride or calcium fluoride for microwave photonics applications. We have fabricated and characterized $5 \mathrm{~mm}$ diameter toroid $\mathrm{MgF}_{2}$ and $12 \mathrm{~mm} \mathrm{CaF}_{2}$ resonators with a good surface roughness $(1 \mathrm{~nm})$. Quality factors better than $3.4 \times 10^{8}$ for magnesium fluoride [7] and $1.4 \times 10^{8}$ for calcium fluoride were measured by cavity ring-down measurements. Even if an optical line can be used as a frequency-selective component in the OEO loop, the use of the mini-resonator leads to a significant reduction of the oscillator size. Further work consists in measuring the phase noise on dedicated instruments developed at the laboratory $[16,17]$. We can also reduce or eliminate sensitivity to vibration by using a refractive index adapter gel. The $Q$ factor of resonators can still be improved by a better polishing of the crystal coupled with other treatments.

\section{Conclusion}

The work performed here is significant in the sense that it appears that the problem of the coupling between a high- $Q$-factor resonator and its external coupler has been theoretically and experimentally improved. The key is to recognize that oscillations of ringing induced by the sweeping of the excitation frequency of an active microsphere are observed. The measured apparent gain is approximately $27 \mathrm{~dB}$ (up to $33.9 \mathrm{~dB}$ in the stationary regime) in a $330 \mathrm{kHz}$ band corresponding in the best case to a high quality factor 


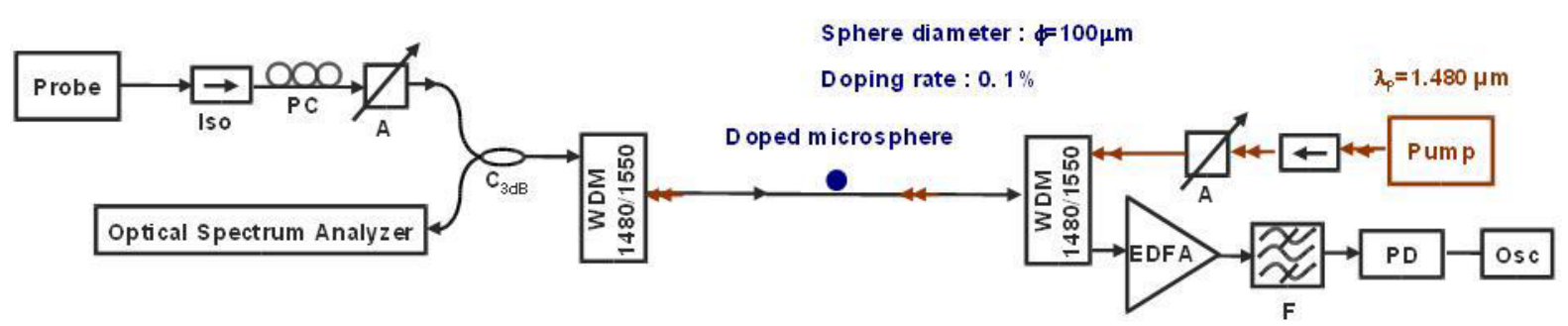

Figure 2. Setup for measuring the selective gain. Probe: extended cavity laser diode with a $150 \mathrm{kHz}$ spectral width, emitting around $1550 \mathrm{~nm}$. Iso: isolator, PC: polarization controller, C: 3dB coupler, WDM: multiplexer 1480/1550, PD: photodetector, EDFA: optical amplifier; F: tunable filter with a $50 \mathrm{pm}$ spectral width, A: optical attenuator, Osc: oscilloscope. 'Pump' is a laser diode emitting around $1480 \mathrm{~nm}$. The diameter of the microsphere is $105 \mu \mathrm{m}$.

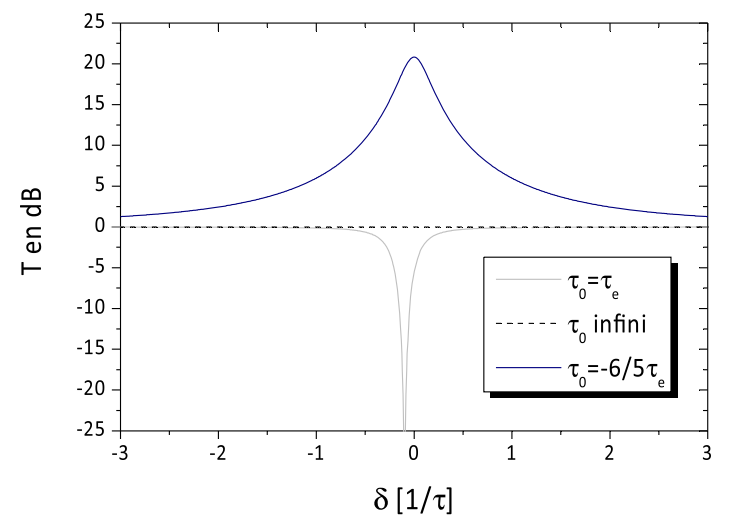

Figure 3. Theoretical spectra obtained for $3 \tau_{0}$.
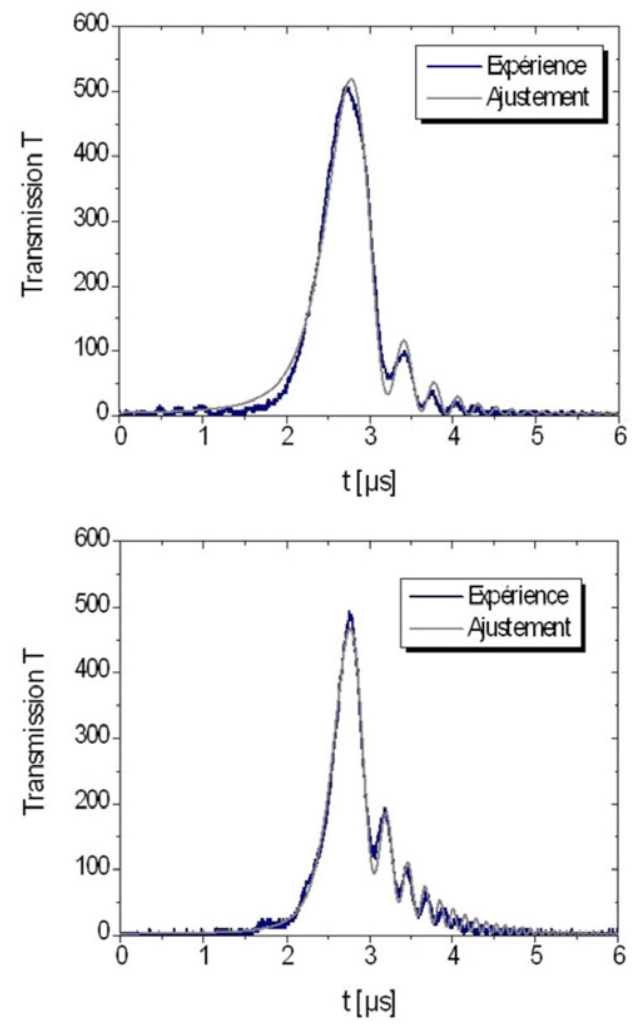

Figure 4. Obtained ringing oscillation profiles for two frequencies $F$ driving the sweeping of the tunable laser, $\mathrm{PS}=125 \mathrm{nW}$, $\mathrm{PP}=7 \mathrm{~mW}$. Top: $F=400 \mathrm{~Hz}$; these values are deduced from the

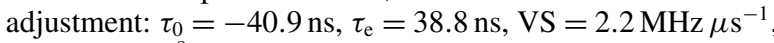
$Q=4.4 \times 10^{8}$ and $T(0)=31.2 \mathrm{~dB}$. Bottom: $F=800 \mathrm{~Hz}$, also deduced from the adjustment: $\tau_{0}=-39.2 \mathrm{~ns}, \tau_{\mathrm{e}}=37.6 \mathrm{~ns}$, $\mathrm{VS}=4.1 \mathrm{MHz} \mu \mathrm{s}^{-1}, Q=5.8 \times 10^{8}$ and $T(0)=33.9 \mathrm{~dB}$.
$Q=5.8 \times 10^{8}$. All of these approaches have been developed to address high-quality factor resonator characterization. Addressing the combination of resonator polishing with other treatments, such as thermal annealing, to improve the surface roughness is still an active area of research to achieve higher $Q$ factors.

\section{Acknowledgment}

We acknowledge the French National Research Agency (ANR) grant no. 'ANR 2010 BLAN 0312'.

\section{References}

[1] Volyanskiy K, Salzenstein P, Tavernier H, Pogurmirskiy M, Chembo Y K and Larger L 2010 Opt. Express 18 22358-63

[2] Vahala K J 2003 Nature 424839

[3] Ilchenko V and Matsko A 2006 IEEE J. Sel. Top. Quantum Electron. 12 15-32

[4] Yao X S and Maleki L 1996 Opt. Lett. 21 483-5

[5] Ilchenko V S, Savchenkov A A, Byrd J, Solomatine I, Matsko A B, Seidel D and Maleki L 2008 Opt. Lett. 33 1569-71

[6] Grudinin I S, Yu N and Maleki L 2009 Opt. Lett. 34 878-80

[7] Tavernier H, Salzenstein P, Volyanskiy K, Chembo Y K and Larger L 2010 IEEE Photon. Technol. Lett. 22 1629-31

[8] Armani D K, Kippenberg T J, Spillane S M and Vahala K J 2003 Nature 421925

[9] Del'Haye P, Schließer A, Arcizet O, Wilken T, Holzwarth R and Kippenberg T J 2007 Nature 450 1214-7

[10] Lissillour F, Messager D, Stéphan G and Féron P 2001 Opt. Lett. 26 1051-3

[11] Dumeige Y, Trebaol S, Ghisa L, Nguyen T K N, Tavernier H and Féron P 2008 J. Opt. Soc. Am. B 25 2073-80

[12] Mortier M, Goldner P, Féron P, Stéphan G M, Xu H and Cai Z P 2003 J. Non-Cryst. Sol. 326-327 505-9

[13] Cai Z P, Xu H Y, Stéphan G, Féron P and Mortier M 2004 Opt. Commun. $229311-5$

[14] He L, Ozdemir S, Xiao Y-F and Yang L 2010 IEEE J. Quantum Electron. 46 1626-33

[15] Trebaol S, Dumeige Y and Féron P 2010 Phys. Rev. A 81043828

[16] Salzenstein P, Cussey J, Jouvenceau X, Tavernier H, Larger L, Rubiola E and Sauvage G 2007 Acta Phys. Pol. A 112 1107-11

[17] Salzenstein P, Pavlyuchenko E, Hmima A, Cholley N, Zarubin M, Galliou S, Chembo Y K and Larger L 2012 Phys. Scr. T149 014025 